\title{
CLINICAL PHARMACOKINETICS OF LOCAL ANAESTHETICS
}

\author{
Alon P. Winnie, Dale A. LaVallee, Buddy Pe Sosa and Khan Z. Masud
}

The PRESENT STUdY was prompted by the very definite clinical impression of the authors that when mepivacaine is used for most supraclavicular techniques of brachial plexus block, the onset of motor blockade precedes the onset of sensory blockade, an observation obviously contrary to the findings of all pharmacologists and physiologists since Gasser and Erlanger. ${ }^{1}$ In order to assess the validity of this observation by a technique that would allow accurate evaluation of the sequence of modality loss in an entirely clinical setting, the authors utilized the subclavian perivascular technique of brachial plexus anaesthesia. This particular technique was selected because with it the injection of a local anaesthetic is made into a closed perineural compartment, at a level where all of the fibers of the brachial plexus are contained in the three large trunks of the plexus, with those fibers destined for distal distribution occupying the center or "core" of each trunk, and those fibers destined for proximal distribution occupying the periphery or "mantle" of each trunk. ${ }^{2}$ Thus, since a single injection is made into a closed space, extra-neural and intra-neural diffusion should be constant factors in determining latency if the distribution evaluated is that of the median nerve, since at this level of injection the fibers that will ultimately comprise the median nerve are deeply and centrally located within each of the three trunks of the plexus.

The present study represents a preliminary study in the search for an ideal model which will allow the controlled study of the pharmacokinetics of local anaesthetic agents in man.

\section{Methods ANd MaterLals}

The present study attempts to compare the anaesthetic activity of the four amide local anaesthetics currently available in the United States in concentrations which are theoretically comparable, as follows: 80 healthy adult patients known to be free from cardiopulmonary disease and neurological disorders and about to undergo surgery involving the upper extremity were divided randomly into four groups, on the basis of which amide local anaesthetic they were about to receive. The patients in Group I received lidocaine 1 per cent, those in Group II received mepivacaine 1 per cent, those in Group III prilocaine I per cent, and those in Group IV bupivacaine 0.25 per cent. No distinction was made as to sex, age, or type of operation, as long as the patient was physiologically an adult and was considered to represent a physical status I. No attempt was made to standardize the premedication, though all patients received an appropriate dose of narcotic along with

Alon P. Winnie, M.D., Dale A. LaVallee, M.D., Buddy Pe Sosa, M.D., and Khan Z. Masud, M.D., Department of Anesthesiology, Abraham Lincoln School of Medicine, University of Illinois Hospitals, 840 South Wood Street, Chicago, Illinois 60612. 


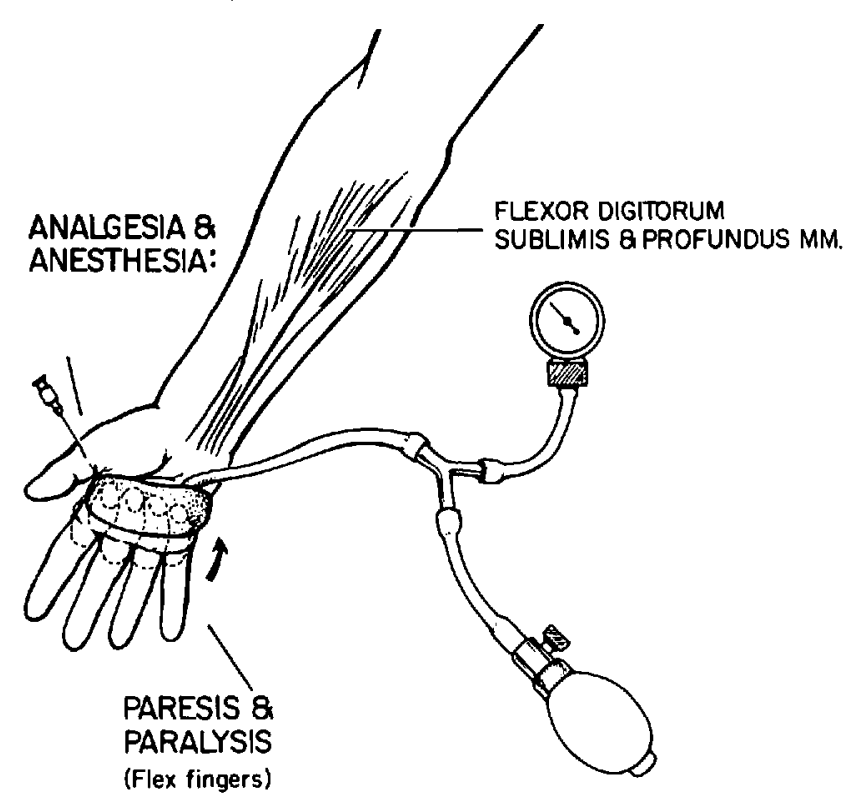

Ficure 1. Experimental method for determining onset and duration of analgesia, anaesthesia, paresis and paralysis (see text).

atropine $0.4 \mathrm{mg}$ approximately 30 to 45 minutes before the anticipated time of block. The subclavian perivascular brachial block was carried out in all patients by the technique described elsewhere by one of the authors, ${ }^{3}$ using a volume of local anaesthetic appropriate for the particular patient, usually a volume in $\mathrm{ml}$ equal to $\frac{12}{2}$ the height in inches. ${ }^{4}$ Following the production of an appropriate paraesthesia, a stop watch was started as the injection of local anaesthetic was begun, to determine the onset and duration of sensory and motor blockade as follows (Figure 1): the onset of sensory blockade was determined by noting the response to pinprick on the thumb in the distribution of the median nerve and the achievement of two endpoints was noted: analgesia was said to exist when the patient no longer perceived the pin as sharp, while anaesthesia was said to exist when the patient did not feel the pin at all. The onset of motor blockade was determined by encircling the palm of the hand with a slightly inflated paediatric blood pressure cuff attached to an anaroid manometer and following the decreasing ability of the patient to compress the cuff by making a fist. The onset of motor blockade, or paresis, was designated as that point when the patient could no longer reach the "pre-block" pressure by compressing the cuff, while complete block, or paralysis, was said to exist when the patient was no longer able to produce any pressure or when the motor block became maximal, as indicated by no further decrease in the pressure the patient could generate. At the point of maximal motor blockade, the patient was asked to elevate the entire arm and the degree to which he was able to do so, together with the amount of pressure he was still able to produce by flexing the fist, determined the intensity of motor blockade, which was classified as follows: the motor block was considered to be complete when a patient was unable to produce any pressure on the manometer with his fingers and if he was unable 


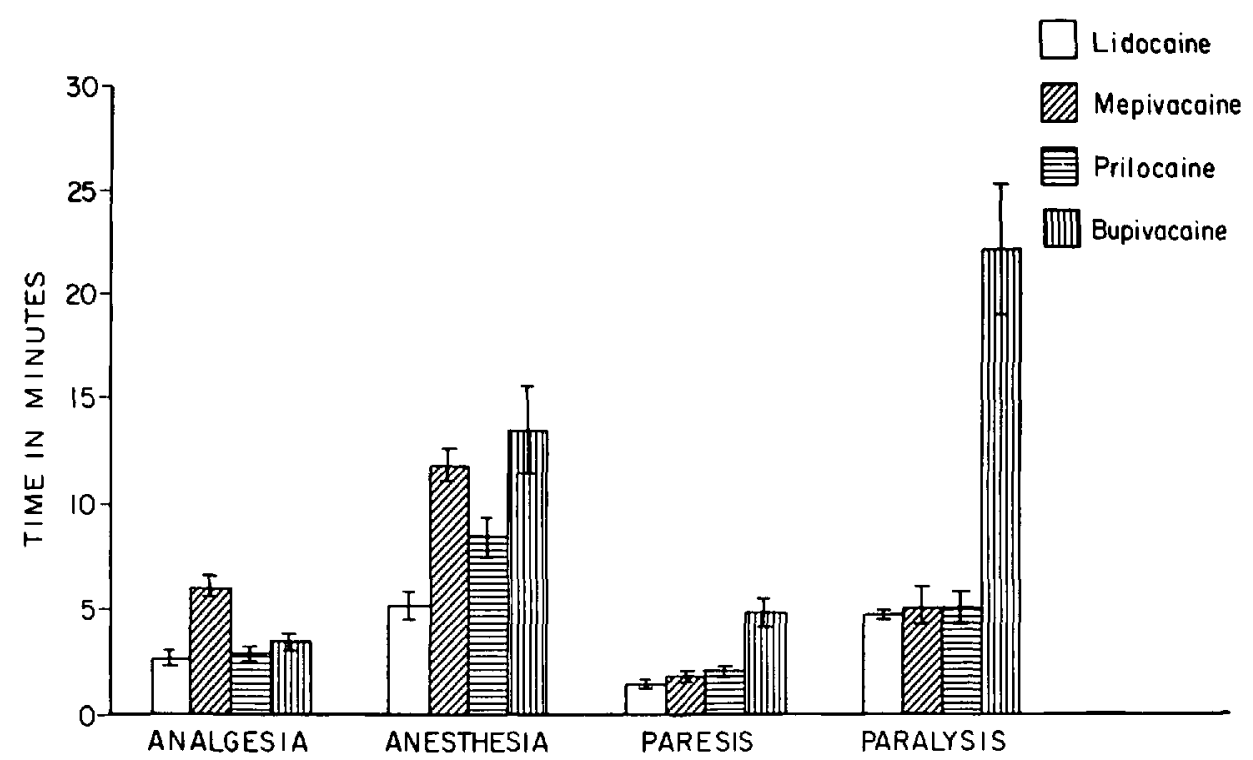

FIcUne 2. Graphic representation of mean values for onset of analgesia, anaesthesia, paresis and paralysis with standard error of the mean.

to elevate the arm at all; the block was considered to be incomplete if the patient was able to produce some pressure on the manometer by flexing his fingers but was unable to elevate the arm at all; and the motor blockade was designated as minimal if the patient could both produce pressure on the manometer and raise the arm off the table.

The duration of analgesia, anaesthesia, paresis, and paralysis, were determined by noting the time required for the function of each modality to return to normal. If during any of the study the block wore off before the completion of the operation so that the duration of the various modalities could not be determined, that particular patient was excluded from the series.

\section{RESULTS}

The data obtained with respect to the onset and duration of analgesia, anaesthesia, paresis, and paralysis are presented in Table I. Figure 2 displays the data with respect to the onset of the various modalities graphically and it may be seen that the onset of analgesia is fastest with lidocaine ( 2.5 minutes) and slowest with mepivacaine (6.0 minutes), with prilocaine and bupivacaine occupying intermediate positions in that order ( 2.8 and 3.3 minutes, respectively). However, while mepivacaine differs statistically from all three of the other agents $(p<0.001)$, none of the others differ from each other significantly with respect to onset of analgesia. In comparing the four agents with respect to the onset of total anaesthesia, it will be noted that while lidocaine and prilocaine still ranked first and second (5.1 and 8.3 minutes, respectively), mepivacaine was third (11.7 minutes) and bupivacaine the slowest in this respect (13.5 mimutes). As far as onset of anaesthesia is con- 


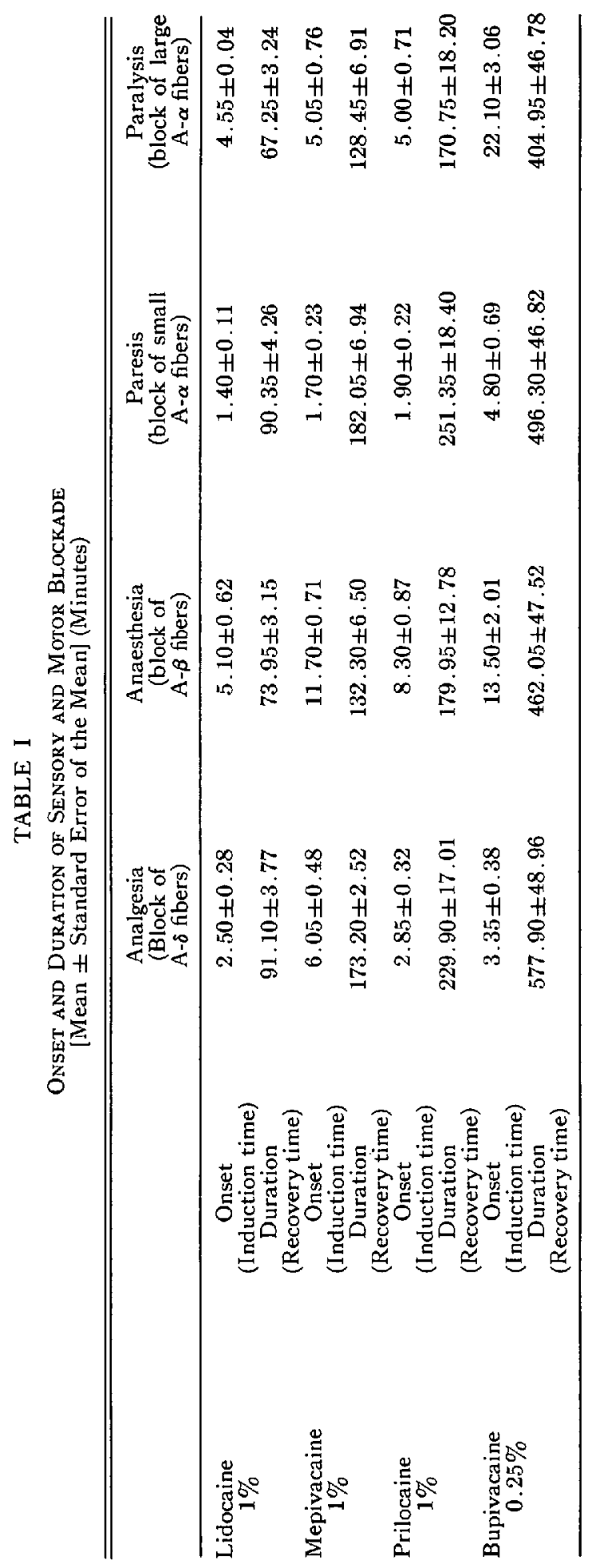




\section{DURATION OF SENSORY \& MOTOR BLOCK}

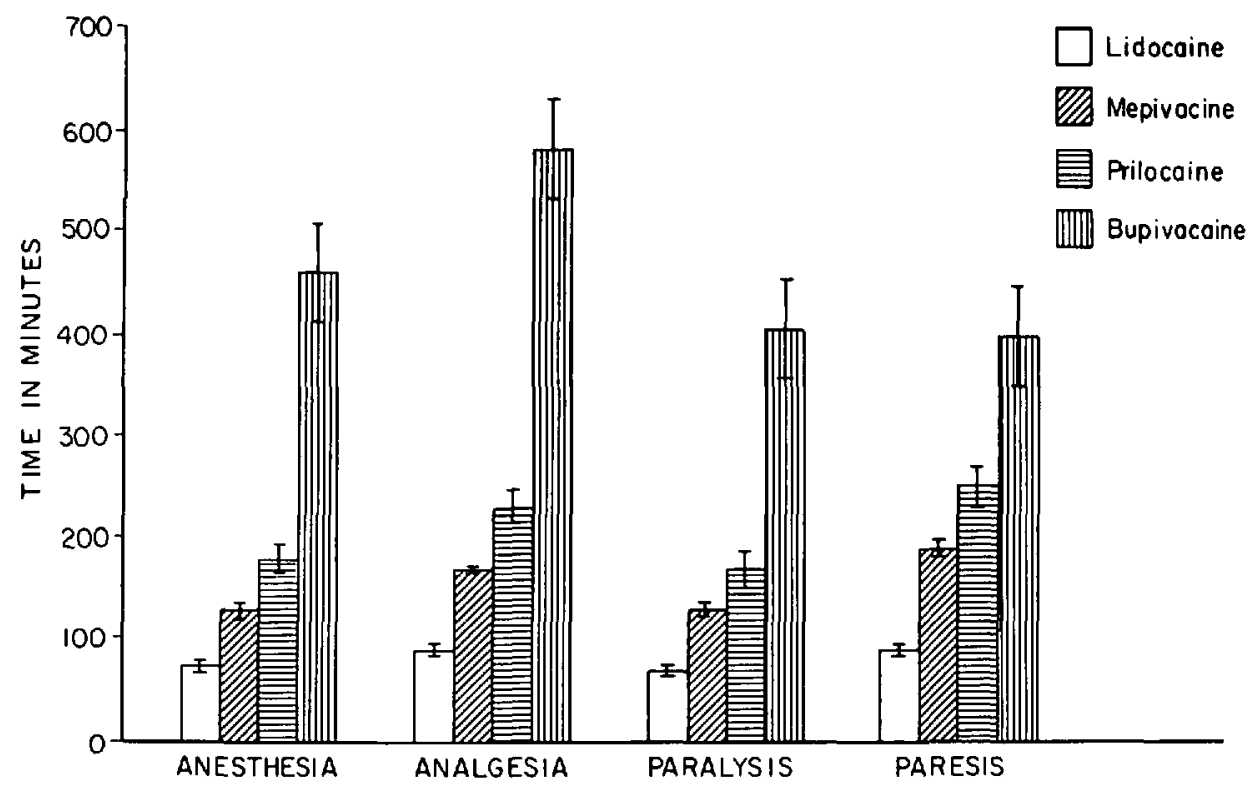

FIgURE 3. Graphic representation of mean values for duration of analgesia, anaesthesia, paresis and paralysis with standard error of the mean.

cerned, all the agents differ significantly from each other $(p<0.01)$ except for bupivacaine and mepivacaine. With respect to the onset of motor blockade, or paresis, and the time to maximal motor blockade, or paralysis, lidocaine was the fastest ( 1.4 and 4.5 minutes, respectively), and bupivacaine the slowest ( 4.8 and 22.0 , respectively), with mepivacaine ( 1.7 and 5.0 minutes), and prilocaine ( 1.9 and 5.0 minutes) being intermediate and virtually equal. Statistically, however, in terms of time to paresis, mepivacaine did not differ significantly from lidocaine or prilocaine, while all the other agents did differ significantly $(\mathrm{p}<0.01)$ and, in terms of paralysis, both mepivacaine and lidocaine did not differ significantly from prilocaine or, in fact, from each other, while the other agents were significantly different $(\mathrm{p}<0.001)$.

Figure 3 displays graphically the recovery data or, in other words, the duration of loss of each modality. With respect to the duration of analgesia and anaesthesia, bupivacaine was the longest acting ( 578 and 462 minutes, respectively) and lidocaine was the shortest acting ( 91 and 74 minutes) with prilocaine ( 230 and 180 minutes) and mepivacaine ( 173 and 132 minutes) being intermediate between the two. All of these differences were statistically highly significant $(p<0.001$ or 0.0001 ). As regards the duration of motor blockade, without respect to the intensity of the blockade, bupivacaine was the longest acting, with paresis lasting $8{ }_{4}^{1 /}$ hours

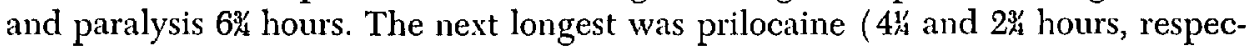
tively), the next was mepivacaine ( 3 and 2 hours) and the shortest acting was lidocaine, with paresis persisting only $1 \frac{1}{2}$ hours and paralysis one hour. Again, all of these durations are significantly different from each other statistically. However, with respect to the intensity of the maximal motor block produced (Figure 4) 
INTENSITY of MOTOR BLOCKADE

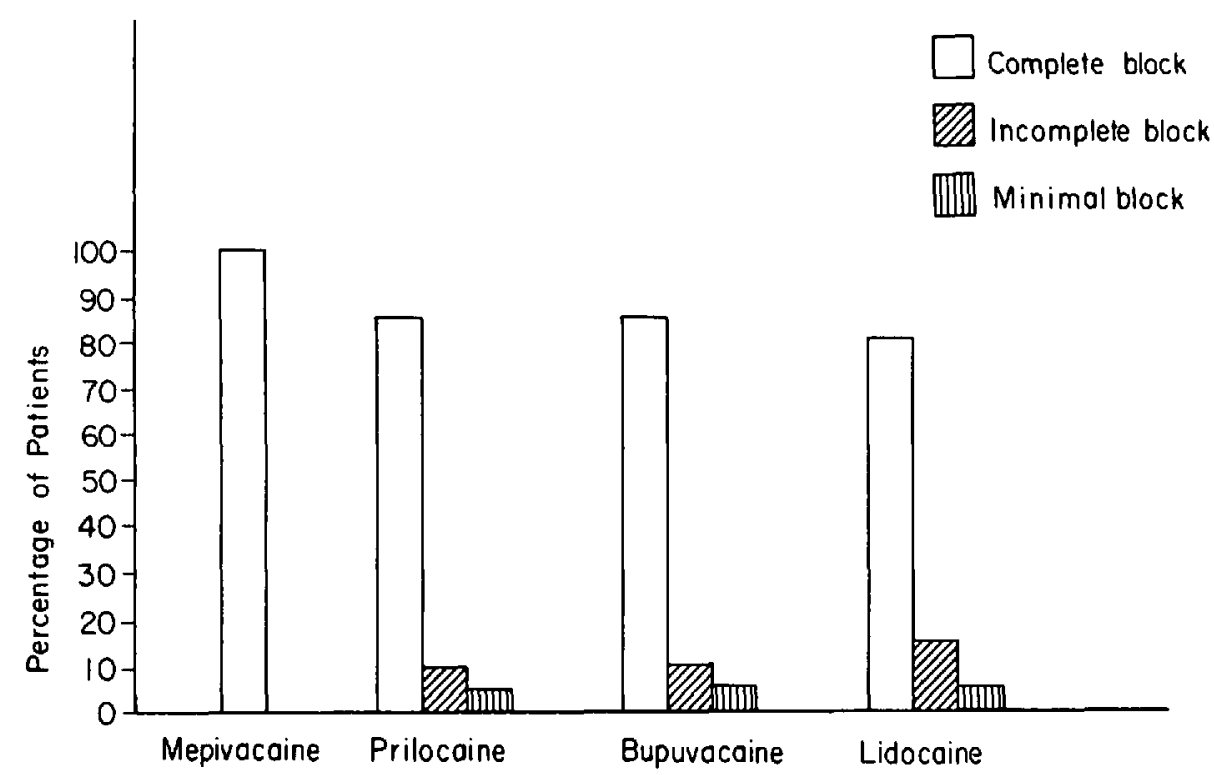

Figure 4. Graphic representation of the intensity of motor blockade produced by each of the four agents.

mepivacaine was superior to all of the others, with 100 per cent of the patients exhibiting complete paralysis with this agent. Prilocaine and bupivacaine were equipotent in this regard, with 85 per cent of the patients exhibiting complete blockade, 10 per cent incomplete blockade, and 5 per cent only minimal blockade. Lidocaine was slightly less potent than these two, with 80 per cent of the patients exhibiting complete blockade, 15 per cent incomplete, and 5 per cent minimal blockade.

Perhaps more significant than the comparative data pertaining to the onset and duration of sensory and motor blockade between the four agents is the sequence in which the various modalities were blocked and the sequence in which they recovered. Figure 5 displays graphically the sequence in which each of the modalities were blocked following the injection of the various local anaesthetic agents, and it becomes immediately apparent that with all agents but bupivacaine, the onset of motor blockade or paresis either precedes or is concomitant with the onset of sensory blockade or analgesia. Furthermore, in Figure 6, which graphically illustrates the sequence in which the various modalities recover, it may be seen that again, with the exception of bupivacaine, motor weakness or paresis lasts as long as or longer than analgesia.

\section{Discussion}

It is almost fifty years since Gasser and Erlanger determined that sensitivity to local anaesthetics is a function of the diameter of a nerve fiber. As a consequence of that concept it has subsequently been shown that the critical sensory concentra- 


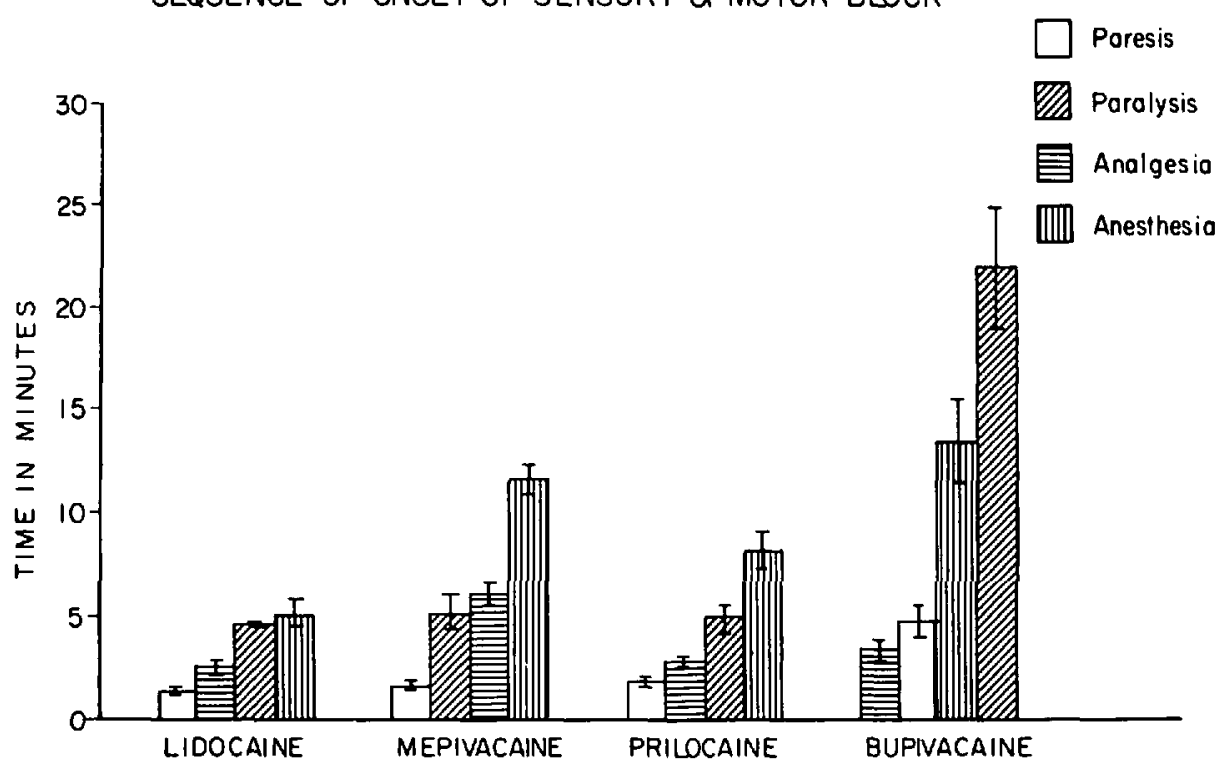

FIgure 5. Graphic representation of the sequence in which each modality was blocked with each agent with standard error of the mean.

SEQUENCE OF RECOVERY OF SENSORY \& MOTOR BLOCK

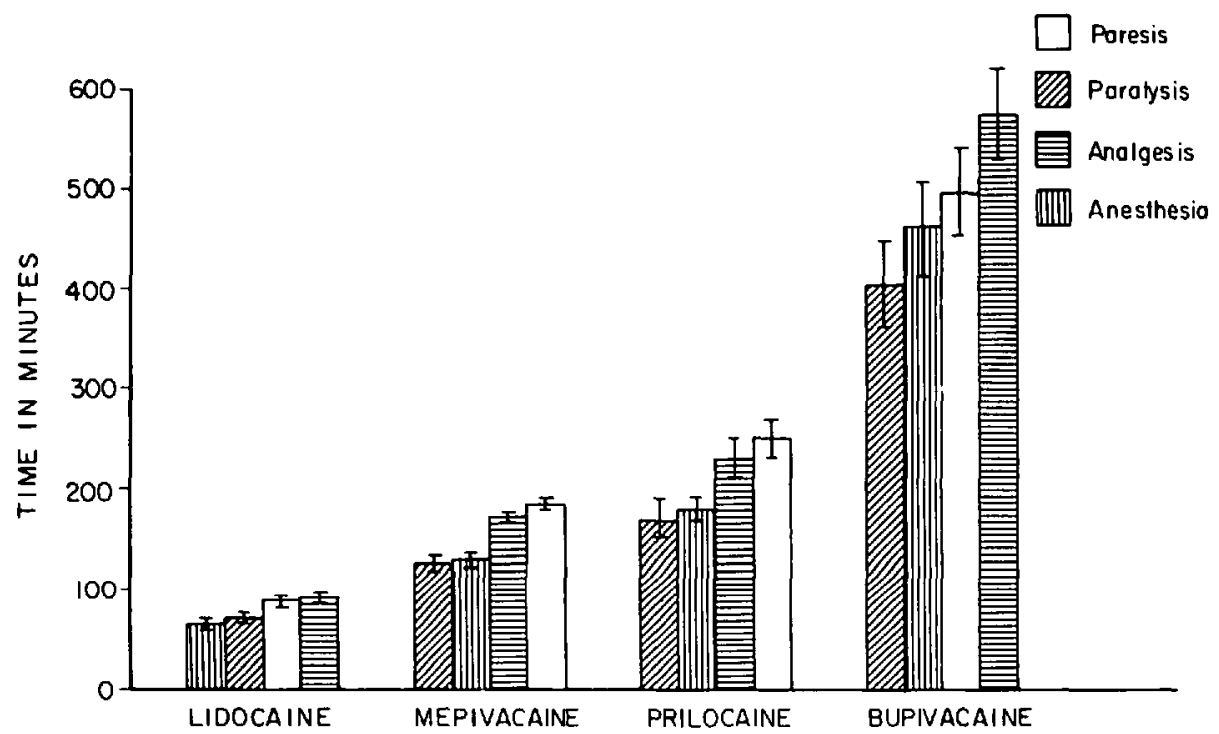

Figure 6. Graphic representation of the sequence in which the various modalities recovered with each agent with standard error of the mean.

tion of a local anaesthetic is lower than the critical motor concentration; that is to say it requires a lower concentration of a local anaesthetic to block sensory fibers than it does to block motor fibers. Thus, in the clinical situation, when a nerve containing both types of fibers is injected with a local anaesthetic agent of a given 


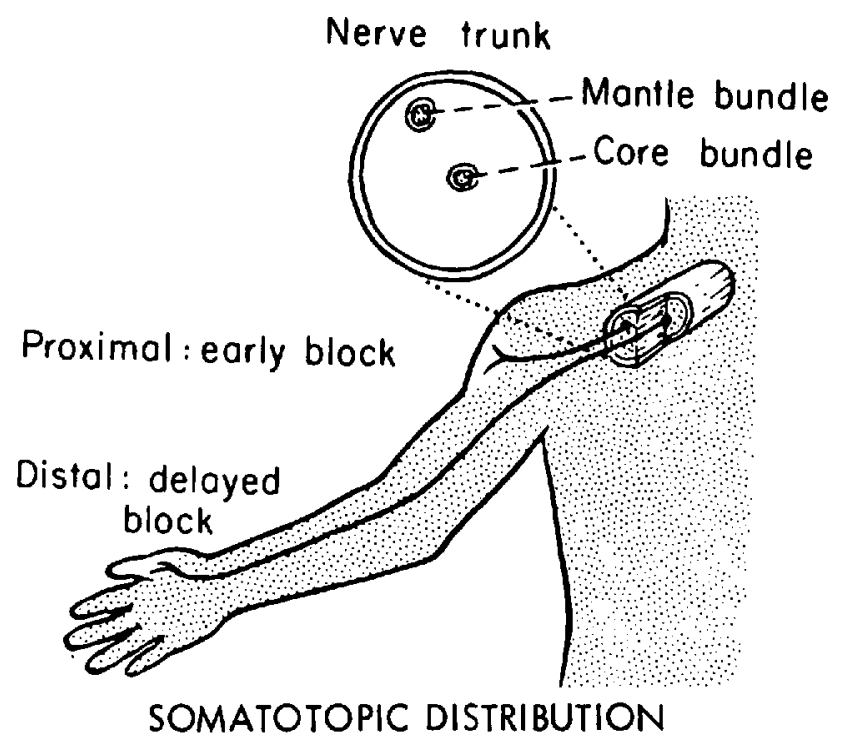

Figure 7. Representation of the somatotopic arrangement of fibers in the trunks of the brachial plexus with those fibers headed for proximal distribution being contained in a mantle (or peripheral) bundle and those headed for distal distribution being contained in a core (or central) bundle. (From de Jong, R.H.: Physiology and Pharmacology of Local Anesthesia, Charles C. Thomas, Springfield, Illinois. With permission of author and publisher.)

concentration, provided that concentration is above the critical motor level, theoretically A- $\delta$ fibers should be blocked first, A- $\beta$ fibers next, and A- $\alpha$ fibers last. That is, analgesia should appear first, followed by anaesthesia, paresis, and paralysis, in that order. However, the results of the present study not only verify our clinical impression that mepivacaine produces motor blockade prior to sensory blockade, but also that prilocaine and lidocaine do likewise.

The answer to this seeming paradox may lie in the difference between the laboratory situation utilized by Gasser and Erlanger and subsequent neurophysiologists and the clinical situation in the present study. Most neurophysiological studies, and certainly those which resulted in the establishment of the relationship between fiber size and sensitivity to local anaesthetics, were carried out using single nerve fiber preparations. In such a preparation there is no delay due to extra-neural diffusion and penetration of fibrous tissue barriers, or even intra-neural diffusion. However, in the clinical situation of the present study, the local anaesthetic was injected around trunks which are extremely large mixed nerves, so that extra-neural diffusion, penetration of the heavy fibrous tissue barriers and intra-neural diffusion become exceedingly important factors in determining the sequence of onset of a block. This is particularly true with our clinical model, since the distribution studied is that of the median nerve which, as pointed out earlier, occupies the core or central portion of each of the three trunks. Since the nerve fibers in a trunk are arranged in such a way (Figure 7) that fibers to or from the proximal regions of the limb travel in the peripheral or mantle bundles, while those to or from the distal regions travel in the core bundles, as a local anaesthetic diffuses from the exterior of the nerve towards the nerve center, the more peripheral fibers should 


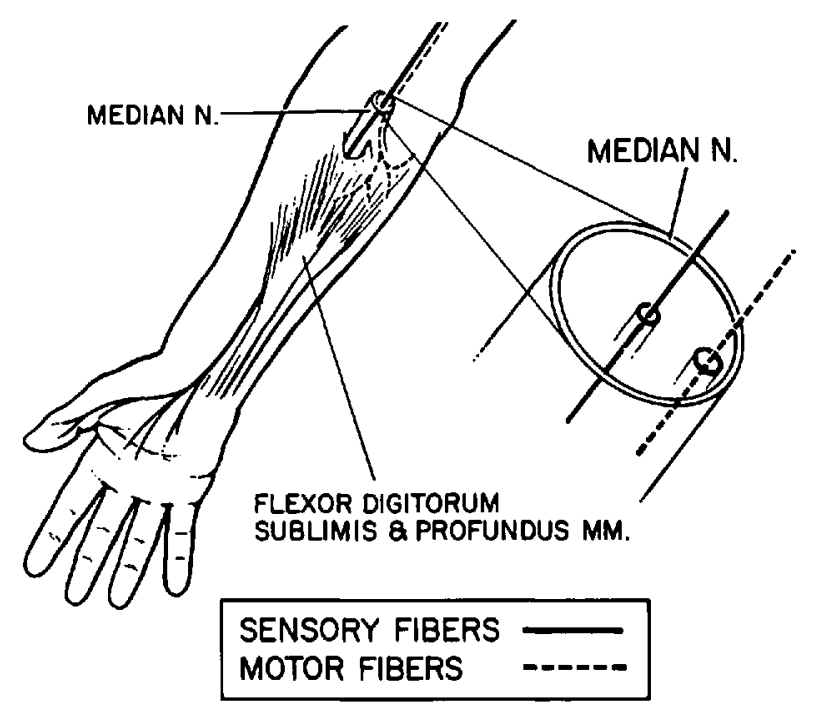

Figure 8. Somatotopic distribution of the fibers within the median nerve, demonstrating the fact that since the motor fibers of the median nerve leave the nerve long before the sensory fibers, motor fibers will occupy a more peripheral position than sensory fibers within the nerve.

be blocked first. ${ }^{2}$ Within the core bundles, which contain the fibers that will comprise the median nerve, there is a similar somatotopic representation, with the more peripheral fibers in the bundle innervating the more proximal portions of nerve distribution and the fibers innervating a slightly more distal distribution being further from the surface or deeper within the core (Figure 8).

Since the motor fibers of the median nerve, which innervates the powerful flexors of the fingers, leave the median nerve just below the elbow, these fibers at the level of the trunks must be located in a position significantly less central than the position of the sensory fibers which terminate in the hand. In other words, in a given core bundle virtually all of the peripheral fibers will be motor, since their distribution is more proximal than the sensory fibers, which will occupy the central portion of the core bundle. Thus as the anaesthetic molecules arrive at the core fibers, they will encounter the more peripheral motor fibers before they reach the more central sensory fibers. Therefore if the concentration of the local anaesthetic agent injected is above the critical motor concentration, the geographical arrangement of the fibers will determine the sequence of blockade, with the onset of motor block occurring before or, at least, simultaneous with the onset of sensory block. However, if the concentration of injected local anaesthetic is barely above, or is below the critical motor level, or if during the course of diffusion it falls below the critical motor level, then the sensory blockade may very well appear first, even though the motor fibers have been bathed with this solution before the sensory fibers. Hence it is not surprising to find in the present study that, with bupivacaine 0.25 per cent, a concentration barely able to produce motor blockade, sensory block precedes motor block, whereas with all three other agents injected in a 1 per cent concentration, motor block precedes sensory.

In short, what this study indicates is that when a local anaesthetic is injected 
next to a large nerve trunk, if the concentration of that anaesthetic is well above the critical motor level, then the geographical arrangement of the nerve fibers within the nerve will determine the sequence in which the various modalities are blocked. On the other hand, if the concentration is borderline with respect to its ability to block motor function, then the geographical arrangement of the nerve fibers within the nerve will be of little consequence. Though the solution reaches the motor nerve first, because of its concentration and the large size of the motor nerve, it is unable to block these fibers, while upon reaching the smaller sensory fibers it is able to produce analgesia and anaesthesia.

While this study verifies and explains our clinical impression that following subclavian perivascular brachial plexus block the onset of motor blockade precedes sensory blockade, it also serves to raise other questions with respect to recovery. According to the currently held concept," after establishment of both motor and sensory blockade, when the extra-neural anaesthetic reservoir has been depleted by diffusion, dispersion, and vascular absorption, the concentration gradient will be reversed and the intra-neural concentration of anaesthetic will begin to decrease. Since the critical motor concentration is higher than the critical sensory concentration, the anaesthetic concentration within the nerve will drop below the critical motor concentration first and only after a further decrease in anaesthetic concentration will it decrease to a level below the critical sensory concentration.

As a result, motor function should recover before sensory function. As may be seen in Figure 3, which displays graphically the sequence of recovery of the four modalities studied for each agent, the duration of paresis either exceeds the duration of analgesia or else does not differ to a statistically significant degree. Studies are currently under way utilizing a refinement of the present model in an attempt to explain this seemingly contradictory finding.

\section{SUMMARY}

The present study has utilized a clinical model to compare the pharmacokinetics of four currently available amide local anaesthetic agents in theoretically equipotent concentrations. In addition to providing comparative data concerning the onset and duration of analgesia, anaesthesia, paresis, and paralysis, it has provided definitive confirmation of the clinical impression that under certain circumstances following the performance of a nerve block, motor blockade may actually precede sensory blockade, and an explanation for this seeming violation of established neurophysiological principles has been postulated. The study has also raised questions concerning the sequence of recovery from motor and sensory blockade which still await explanation.

\section{RÉSUMÉ}

La présente étude fait appel à un modèle clinique, pour comparer la pharmacocynétique de quatre anesthésiques locaux, d'usage courant, à des concentrations et des puissances équivalentes.

Grâce à ce modèle, nous avons pu recueillir des données précises sur le début et 
la durée de l'analgésie produite, et sur la qualité de l'anesthésie (parésies, paralysies ). L'impression clinique que le bloc moteur précède parfois le bloc sensitif a été confirmée. Une hypothèse a été soulevée pour expliquer ce phénomène. Cette étude soulève encore de nombreuses questions quant à la séquence de récupération des blocs moteur et sensitif.

\section{REFERENCES}

1. Gasser, H.S. \& Erlanger, J. The role of fiber size in the establishment of a nerve block by pressure or cocaine. Amer. J. Physiol. 88: 58 (1929).

2. De Jong, R.H. Physiology and Pharmacology of Local Anaesthesia, Springfield: Charles C. Thomas (1970).

3. Winme, A.P. \& Collins, V.J. The subclavian perivascular technique of brachial plexus anesthesia. Anesthesiology 25: 353 (1964).

4. Winnie, A.P. Interscalene brachial plexus block. Anesth. Analg. 49: 455 (1970). 